\title{
Structural and elemental analysis of presolar silicon carbide grains
}

\author{
RHONDA. M. STROUD ${ }^{1 *}$, SHERYL A.SINGERLING ${ }^{2}$, NAN \\ LIU $^{3}$, CONEL M. O’D. ALEXANDER ${ }^{4}$ AND LARRY R. \\ NITTLER $^{4}$
}

${ }^{1}$ U.S. Naval Research Laboratory, Washington, DC 20375

(*correspondence: rhonda.stroud@nrl.navy.mil)

${ }^{2}$ Nova Research Inc., Alexandria, VA 22308

${ }^{3}$ Washington University, St. Louis, MO 63130

${ }^{4}$ Carnegie Institution of Washington, Washington, DC 20015

Silicon carbide dust grains from a range of classes of ancient stars are preserved in primitive meteorites. These grains provide a record of the condensation conditions in their progenitor stellar sources, and subsequent processing histories in the interstellar medium, which can be revealed through direct laboratory analyses of the bona fide stardust. Important outstanding questions include the range of structural and compositional variations specific to different stellar origins, especially for the more rare grains from lowmetallicity AGB and non-AGB stars. Our recent work has shown that confocal Raman spectroscopy coupled with energy dispersive $\mathrm{x}$-ray spectroscopy (EDS) in a scanning electron microscope (SEM) can be useful for non-destructive identification of grains with unusual elemental, isotopic and/or structural features, which are then targets for more detailed investigation by transmission electron microscopy (TEM) [1].

We selected grains for focused ion beam sectioning and TEM analysis from among those reported in [1]. To date we have sectioned nine grains, including 5 MS grains (AGB), 3 $\mathrm{X}$ grains (type II supernova), and $1 \mathrm{Y}$ grain (low-metallicity AGB). Our results confirm that observed deviations in the Raman spectral features in the reference TO peak position for the $3 \mathrm{C}$ polytype $\left(\sim 800 \mathrm{~cm}^{-1}\right)$ are correlated with variations in the polytype and crystal quality. In addition, sub-grains were visible in Z-contrast scanning TEM images of 6 out of 7 grains imaged, with most grains containing multiple subgrains. Small voids, which appear to be primary growth features with no detectable trapped volatiles, are also common. We will present the detailed TEM results, and discuss the implications for constraining the condensation and processing histories in different stellar environments, and for interpretation of optical astronomical observations of $\mathrm{SiC}$ dust.

[1] Liu N. et al. Meteorit. Planet. Sci. 52 (2017) 2550-2569. 